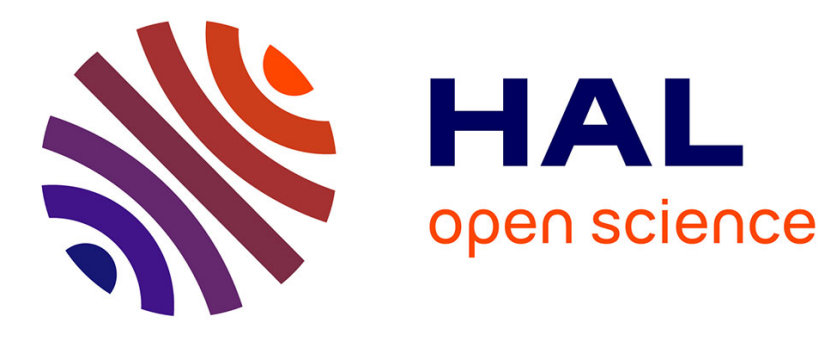

\title{
Cyclicity in Dirichlet type spaces
}

Karim Kellay, Florian Le Manach, Mohamed Zarrabi

\section{To cite this version:}

Karim Kellay, Florian Le Manach, Mohamed Zarrabi. Cyclicity in Dirichlet type spaces. Complex Analysis and Spectral Theory. 181-193, Contemp. Math., 743, Amer. Math. Soc., Providence, RI, [2020], May 2018, Québéc, Canada. pp.181-193. hal-01941825v2

\section{HAL Id: hal-01941825 \\ https://hal.science/hal-01941825v2}

Submitted on 7 May 2019

HAL is a multi-disciplinary open access archive for the deposit and dissemination of scientific research documents, whether they are published or not. The documents may come from teaching and research institutions in France or abroad, or from public or private research centers.
L'archive ouverte pluridisciplinaire $\mathbf{H A L}$, est destinée au dépôt et à la diffusion de documents scientifiques de niveau recherche, publiés ou non, émanant des établissements d'enseignement et de recherche français ou étrangers, des laboratoires publics ou privés. 


\title{
CYCLICITY IN DIRICHLET TYPE SPACES
}

\author{
K. KELLAY, F. LE MANACH \& M. ZARRABI \\ Dedicated to Thomas Ransford on the occasion of his 60th birthday.
}

\begin{abstract}
We study cyclicity in the Dirichlet type spaces for outer functions whose zero
\end{abstract} set is countable.

\section{INTRODUCTION AND MAIN RESULT}

Let $X$ be a Banach space of functions holomorphic in the open unit disk $\mathbb{D}$, such that the shift operator $S: f(z) \rightarrow z f(z)$ is a continuous map from $X$ into itself. Given $f \in X$, we denote by $[f]_{X}$ the smallest closed $S$-invariant subspace of $X$ containing $f$, namely

$$
[f]_{X}=\overline{\{p f: p \text { is a polynomial }\}} .
$$

We say that $f$ is cyclic in $X$ if $[f]_{X}=X$.

The problem of cyclic vectors in the Dirichlet spaces goes back to the work of Beurling and Carleson (see $[\mathrm{B}, \mathrm{C}]$ ). The classical Dirichlet space $\mathcal{D}$ consists of holomorphic functions on the unit disc whose derivatives are square integrable. While Beurling characterizes cyclic vectors in the Hardy space $H^{2}$, the problem of characterizing the cyclic vectors in the Dirichlet space $\mathcal{D}$ is much more difficult. Beurling's theorem says that the cyclic vectors in $H^{2}$ are the outer functions. On the other hand we know that there are outer functions in the Dirichlet space which are not cyclic in $\mathcal{D}$. In fact, the cyclicity of such a function depends on the distribution of the zeros of the radial limit $f^{*}$ of $f$ on the unit circle. The Brown-Shields conjecture [HS] claims that $f \in \mathcal{D}$ is cyclic iff $f$ is an outer function and the set of all zeros of $f^{*}$ is a set of logarithmic capacity zero. A partial (positive) answer to this conjecture was given in [EKR2, EKR1]. We mention the results of Beurling [B] about the boundary behavior for the functions of the Dirichlet spaces: if $f \in \mathcal{D}$ we write $f^{*}(\zeta)=\lim _{r \rightarrow 1-} f(r \zeta)$, then the radial limit $f^{*}$ exists -q.e on $\mathbb{T}$, that is $f^{*}$ exists outside a set of capacity logarithmic zero. As a consequence of a weak-type inequality the invariant subspace $\mathcal{D}_{E}$ defined by

$$
\mathcal{D}_{E}=\left\{f \in \mathcal{D},\left.f^{*}\right|_{E}=0 \text { q.e. }\right\}
$$

2000 Mathematics Subject Classification. Primary 46E22; Secondary 31A05, 31A15, 31A20, 47B32.

Key words and phrases. Dirichlet spaces, Bergman spaces, Smirnov space, cyclic vectors.

The research of the first author is partially supported by by the project ANR-18-CE40-0035 and by the Joint French-Russian Research Project PRC CNRS/RFBR,2017-2019. 
is closed in $\mathcal{D}$. Carleson in $[\mathrm{C}]$ proved that for every closed subset $E$ of the unit circle which has zero logarithmic capacity, there exists a cyclic function in $\mathcal{D}$ which vanishes on E.

We denote by $A(\mathbb{D})$ the disc algebra. Hedenmalm and Shields showed in $[\mathrm{HS}]$ that if $f \in \mathcal{D} \cap A(\mathbb{D})$ is an outer function and $\mathcal{Z}\left(f^{*}\right)=\left\{\zeta \in \mathbb{T}: f^{*}(\zeta)=0\right\}$, the zero set of $f^{*}$, is countable then $f$ is cyclic in $\mathcal{D}$. Richter and Sundberg in [RS1] improve this result by showing that if $f \in \mathcal{D}$ is outer and $\underline{\mathcal{Z}}(f)=\left\{\zeta \in \mathbb{T}: \liminf _{z \rightarrow \zeta}|f(z)|=0\right\}$ is countable then $f$ is cyclic in $\mathcal{D}$. When the set of zeros of $f^{*}$ is not countable, see [EKMR, EKR1, EKR2] in the case of the classical Dirichlet space $\mathcal{D}_{0}^{2}$ and [EKR3] in the case of $\mathcal{D}_{\alpha}^{2}, 0<\alpha<1$ for further results on cyclicity in that context.

In this paper we are interested in cyclicity, in more general Dirichlet spaces, of outer functions such that the zero set is countable. We now introduce some notations. The Dirichlet/Besov space $\mathcal{D}_{\alpha}^{p}$ with $p \geq 1$ and $\alpha>-1$ is given by

$$
\mathcal{D}_{\alpha}^{p}=\left\{f \in \operatorname{Hol}(\mathbb{D}):\|f\|_{\mathcal{D}_{\alpha}^{p}}^{p}=|f(0)|^{p}+\int_{\mathbb{D}}\left|f^{\prime}(z)\right|^{p} \mathrm{dA}_{\alpha}(z)<\infty\right\} .
$$

where $\mathrm{dA}_{\alpha}$ denotes the finite measure on the unit disc $\mathbb{D}$ given by

$$
\mathrm{dA}_{\alpha}(z):=(1+\alpha)\left(1-|z|^{2}\right)^{\alpha} \mathrm{dA}(z)
$$

and $\mathrm{dA}(z)=\mathrm{d} x \mathrm{~d} y / \pi$ stands for the normalized area measure on $\mathbb{D}$. If $p=2$ and $\alpha=1$, then $\mathcal{D}_{1}^{2}$ is the Hardy space $\mathrm{H}^{2}$ and the classical Dirichlet space corresponds to $p=2$ and $\alpha=0, \mathcal{D}_{0}^{2}=\mathcal{D}$. The following theorem is the main result of this paper.

Theorem. Let $p>1$ be such that $\alpha+1<p \leq \alpha+2$ and let $f \in \mathcal{D}_{\alpha}^{p} \cap A(\mathbb{D})$. If $f$ is outer and $\mathcal{Z}(f)$ is countable, then $f$ is cyclic in $\mathcal{D}_{\alpha}^{p}$.

Notice that when $1<p<\alpha+1, H^{p}(\mathbb{D})$ is continuously embedded in $\mathcal{D}_{\alpha}^{p}$ and every outer function $f \in H^{p}(\mathbb{D})$ is cyclic for $\mathcal{D}_{\alpha}^{p}$ (Proposition 3.1). On the other hand when $p>\alpha+2$ then every function which vanishes at least at one point is not cyclic in $\mathcal{D}_{\alpha}^{p}$.

The method used for the proof of Theorem 3.10 is inspired by that of the Hedenmalm and Shields [HS] in the case of the classical Dirichlet space and the paper [EKR2] .

Throughout the paper, we use the following notations:

- $A \lesssim B$ means that there is an absolute constant $C$ such that $A \leq C B$.

- $A \asymp B$ if both $A \lesssim B$ and $B \lesssim A$ hold.

\section{Dirichlet SpaCe AND DUAlity}

The Bergman spaces $\mathcal{A}_{\alpha}^{p}$ with $p \geq 1, \alpha>-1$ are given by

$$
\mathcal{A}_{\alpha}^{p}(\mathbb{D})=\left\{f \in \operatorname{Hol}(\mathbb{D}),\|f\|_{\mathcal{A}_{\alpha}^{p}}^{p}=\int_{\mathbb{D}}|f(z)|^{p} \mathrm{dA}_{\alpha}(z)<\infty\right\} .
$$


We define the Bergman spaces $\mathcal{A}_{\alpha}^{p}\left(\mathbb{D}_{e}\right)$ on the exterior disk $\mathbb{D}_{e}=(\mathbb{C} \cup\{\infty\}) \backslash \overline{\mathbb{D}}$ with $p \geq 1$ and $\alpha>-1$ by

$$
\mathcal{A}_{\alpha}^{p}\left(\mathbb{D}_{e}\right)=\left\{g \in \operatorname{Hol}\left(\mathbb{D}_{e}\right), g(\infty)=0 \text { and }\|g\|_{\mathcal{A}_{\alpha}^{p}}^{p}=\int_{\mathbb{D}_{e}}|g(z)|^{p} \frac{\left(|z|^{2}-1\right)^{\alpha}}{|z|^{4-p+2 \alpha}} \mathrm{dA}(z)<\infty\right\} .
$$

Note that $\mathcal{A}_{\alpha}^{p}(\mathbb{D})$ and $\mathcal{A}_{\alpha}^{p}\left(\mathbb{D}_{e}\right)$ are isometrically isomorphic via the isometry $R: f \mapsto R f$ defined on $\mathcal{A}_{\alpha}^{p}(\mathbb{D})$ by

$$
R f(z)=\frac{1}{z} \overline{f\left(\frac{1}{\bar{z}}\right)}, \quad z \in \mathbb{D}_{e} .
$$

Indeed, by the variable change $z \mapsto 1 / \bar{z}$,

$$
\int_{\mathbb{D}}|f(z)|^{p} \mathrm{dA}_{\alpha}(z)=\int_{\mathbb{D}_{e}}|\overline{f(1 / \bar{z})} / z|^{p} \frac{\left(|z|^{2}-1\right)^{\alpha}}{|z|^{4-p+2 \alpha}} \mathrm{dA}(z)
$$

Futhermore if $f=\sum_{n \geq 0} a_{n} z^{n} \in \mathcal{A}_{\alpha}^{p}(\mathbb{D})$ then by $(2.1)$

$$
R f(z)=\sum_{n=0}^{\infty} \frac{\overline{a_{n}}}{z^{n+1}}, \quad z \in \mathbb{D}_{e} .
$$

Denote by $S$ the shift operator on $\mathcal{A}_{\alpha}^{p}(\mathbb{D})$ for $p \geq 1$ and $\alpha>-1$, that is the multiplication by z on $\mathcal{A}_{\alpha}^{p}(\mathbb{D})$. Let $S^{*}$ denote the backward shift, that is

$$
S^{*} f(z)=\frac{f(z)-f(0)}{z} .
$$

Notice that $S^{*}$ is continuous on $\mathcal{A}_{\alpha}^{p}(\mathbb{D})$ for $p \geq 1$ and $\alpha>-1$. Indeed, for $f \in \mathcal{A}_{\alpha}^{p}(\mathbb{D})$ we get by subharmonicity ([HKZ, proposition 1.1]) that

$$
\left|\frac{f(z)-f(0)}{z}\right| \leq \sup _{|w| \leq 1 / 2}\left|f^{\prime}(w)\right| \lesssim\|f\|_{\mathcal{A}_{\alpha}^{p}(\mathbb{D})}, \quad|z|<1 / 2 .
$$

Since $f \mapsto f(0)$ is continuous on $\mathcal{A}_{\alpha}^{p}(\mathbb{D})$ ([HKZ, proposition 1.1]), we have

$$
\begin{aligned}
\left\|S^{*} f\right\|_{\mathcal{A}_{\alpha}^{p}}^{p} & \leq \int_{|z| \leq 1 / 2}\|f\|_{\mathcal{A}_{\alpha}^{p}}^{p} \mathrm{dA}_{\alpha}(z)+2^{p} \int_{1 / 2<|z|<1}|f(z)-f(0)|^{p} \mathrm{dA}_{\alpha}(z) \\
& \lesssim\|f\|_{\mathcal{A}_{\alpha}^{p}(\mathbb{D})}^{p}+\|f-f(0)\|_{\mathcal{A}_{\alpha}^{p}(\mathbb{D})}^{p} \\
& \lesssim\|f\|_{\mathcal{A}_{\alpha}^{p}(\mathbb{D})} .
\end{aligned}
$$

From now, we suppose that $p>1$ and we denote by $q=\frac{p}{p-1}$.

Lemma 2.1. Suppose that $-1<\alpha<p-1$. Then $\langle\cdot, \cdot\rangle$ defined on $\mathcal{D}_{\alpha}^{p} \times \mathcal{A}_{-\alpha q / p}^{q}(\mathbb{D})$ by

$$
\langle f, g\rangle=\int_{\mathbb{D}} f^{\prime}(z) \overline{S^{*} g(z)} d A(z)+f(0) \overline{g(0)}, \quad f \in \mathcal{D}_{\alpha}^{p}, g \in \mathcal{A}_{-\alpha q / p}^{q}(\mathbb{D}),
$$

is linear on the left, anti-linear on the right and

$$
|\langle f, g\rangle| \lesssim\|f\|_{\mathcal{D}_{\alpha}^{p}}\|g\|_{\mathcal{A}_{-\alpha q / p}^{q}(\mathbb{D})} .
$$


Proof. Since $-\alpha q / p>-1,(f, g) \rightarrow\langle f, g\rangle$ is well defined. Clearly this map is linear on the left and antilinear on the right. It is therefore sufficient to show that

$$
|\langle f, g\rangle| \lesssim\|f\|_{\mathcal{D}_{\alpha}^{p}}\|g\|_{\mathcal{A}_{-\alpha q / p}^{q}(\mathbb{D})} \cdot
$$

Using Hölder's inequality and the fact that the maps $S^{*}$ and $f \mapsto f(0)$ are continuous on the space $\mathcal{A}_{-\alpha q / p}^{q}(\mathbb{D})$, we get

$$
\begin{aligned}
|\langle f, g\rangle| & \leq \int_{\mathbb{D}}\left|f^{\prime}(z)\right| \frac{\left(1-|z|^{2}\right)^{\alpha / p}}{\left(1-|z|^{2}\right)^{\alpha / p}}\left|\overline{S^{*} g(z)}\right| d A(z)+|f(0) \overline{g(0)}| \\
& \leq\left(\int_{\mathbb{D}}\left|f^{\prime}(z)\right|^{p} \mathrm{dA}_{\alpha}(z)\right)^{1 / p}\left(\int_{\mathbb{D}}\left|S^{*} g(z)\right|^{q} \mathrm{~d} \mathrm{~A}_{-\alpha q / p}\right)^{1 / q}+|f(0)||g(0)| \\
& \leq\|f\|_{\mathcal{D}_{\alpha}^{p}}\left\|S^{*} g\right\|_{\mathcal{A}_{-\alpha q / p}^{q}(\mathbb{D})}+|f(0)||g(0)| \\
& \lesssim\|f\|_{\mathcal{D}_{\alpha}^{p}}\|g\|_{\mathcal{A}_{-\alpha q / p}^{q}(\mathbb{D})} .
\end{aligned}
$$

The previous lemma shows that $\langle\cdot, \cdot\rangle$ defines a duality between $\mathcal{D}_{\alpha}^{p}$ and $\mathcal{A}_{-\alpha q / p}^{q}(\mathbb{D})$. The following result shows that $\mathcal{A}_{-\alpha q / p}^{q}(\mathbb{D})$ can be identified as the dual of $\mathcal{D}_{\alpha}^{p}$.

Proposition 2.2. Let $p>1$ and $-1<\alpha<p-1$. The dual of $\mathcal{D}_{\alpha}^{p}$, noted by $\mathcal{D}_{\alpha}^{p^{\prime}}$, is isomorphic to $\mathcal{A}_{-\alpha q / p}^{q}(\mathbb{D})$.

Proof. We will show that the mapping $g \mapsto\langle\cdot, g\rangle$ is an isomorphism of $\mathcal{A}_{-\alpha q / p}^{q}(\mathbb{D})$ in $\mathcal{D}_{\alpha}^{p \prime}$, the dual of $\mathcal{D}_{\alpha}^{p}$. This mapping is well defined, antilinear, continuous and injective. Let's show that it's surjective. Take $L$ in $\mathcal{D}_{\alpha}^{p \prime}$. For all $f \in \mathcal{A}_{\alpha}^{p}(\mathbb{D})$, we consider $F$ the primitive of $f$ on $\mathbb{D}$ such that $F(0)=0$. It's easy to see that $F \in \mathcal{D}_{\alpha}^{p}$. We define the mapping $L_{0}$ on $\mathcal{A}_{\alpha}^{p}(\mathbb{D})$ by $L_{0}(f)=L(F)$. Thus $L_{0}$ belong to the dual of $\mathcal{A}_{\alpha}^{p}(\mathbb{D})$, since

$$
\left|L_{0}(f)\right|=|L(F)| \leq\|L\|\|F\|_{\mathcal{D}_{\alpha}^{p}}=\|L\|\|f\|_{\mathcal{A}_{\alpha}^{p}} .
$$

By the Hahn-Banach theorem, $L_{0}$ extends to $L_{\alpha}^{p}(\mathbb{D})=L^{p}\left(\mathbb{D}, \mathrm{dA}_{\alpha}\right)$ in a continuous linear form $\widetilde{L_{0}}$. By the Riesz representation theorem, there exists $\psi_{0} \in L_{-\alpha q / p}^{p}(\mathbb{D})=L_{\alpha}^{p}(\mathbb{D})^{\prime}$ such that for any $g \in L_{\alpha}^{p}(\mathbb{D})$,

$$
\widetilde{L_{0}}(g)=\int_{\mathbb{D}} g(z) \overline{\psi_{0}}(z) d A(z)
$$

Let $P$ be the linear map defined by

$$
P: f \mapsto\left(z \mapsto \int_{\mathbb{D}} \frac{f(w)}{(1-z \bar{w})^{2}} d A(w)\right)
$$

According to $\left[\mathrm{HKZ}\right.$, Theorem 1.10], $P$ is a bounded projection from $L_{\gamma}^{s}(\mathbb{D})$ onto $\mathcal{A}_{\gamma}^{s}(\mathbb{D})$ for $\gamma<s-1$ which is the case when $(s, \gamma)=(p, \alpha)$ and $(s, \gamma)=(q,-\alpha q / p)$. Set $\psi=P\left(\psi_{0}\right) \in$ 
$\mathcal{A}_{-\alpha q / p}^{q}(\mathbb{D})$. So for $f \in \mathcal{A}_{\alpha}^{p}(\mathbb{D})$, we get

$$
\begin{aligned}
L_{0}(f)=\widetilde{L_{0}}(f) & =\int_{\mathbb{D}} f(z) \overline{\psi_{0}}(z) d A(z) \\
& =\int_{\mathbb{D}} \int_{\mathbb{D}} \frac{f(w)}{(1-z \bar{w})^{2}} \overline{\psi_{0}}(z) d A(w) d A(z) \\
& =\int_{\mathbb{D}} f(w) \int_{\mathbb{D}} \frac{\psi_{0}(z)}{(1-w \bar{z})^{2}} d A(z) d A(w) \\
& =\int_{\mathbb{D}} f(w) \overline{\psi(w)} d A(w) .
\end{aligned}
$$

Thus we showed that there is $\psi \in \mathcal{A}_{-\alpha q / p}^{q}(\mathbb{D})$ such that for any $F \in \mathcal{D}_{\alpha}^{p}$ with $F(0)=0$, we have

$$
L(F)=\int_{\mathbb{D}} F^{\prime}(z) \overline{\psi(z)} d A(z) .
$$

Set $\varphi(z)=z \psi(z)+\overline{L(1)} \in \mathcal{A}_{-\alpha q / p}^{q}(\mathbb{D})$. We have $S^{*} \varphi=\psi$. Hence for $h \in \mathcal{D}_{\alpha}^{p}$

$$
\begin{aligned}
L(h) & =L(h-h(0))+L(h(0)) \\
& =\int_{\mathbb{D}} h^{\prime}(z) \overline{\psi(z)} d A(z)+h(0) L(1) \\
& =\int_{\mathbb{D}} h^{\prime}(z) \overline{S^{*} \varphi(z)} d A(z)+h(0) \overline{\varphi(0)}=\langle h, \varphi\rangle .
\end{aligned}
$$

This shows that the mapping $g \mapsto\langle\cdot, g\rangle$ is surjective and defines an isomorphism from $\mathcal{A}_{-\alpha q / p}^{q}(\mathbb{D})$ onto $\mathcal{D}_{\alpha}^{p \prime}$.

Remarks. If $p>1$ and $\alpha<p-1$, the dual of $\mathcal{D}_{\alpha}^{p}$ is identified as $\mathcal{A}_{-\alpha q / p}^{q}(\mathbb{D})$. Also the spaces $\mathcal{A}_{-\alpha q / p}^{q}(\mathbb{D})$ and $\mathcal{A}_{-\alpha q / p}^{q}\left(\mathbb{D}_{e}\right)$ are isomorphic, so we can identify the dual of $\mathcal{D}_{\alpha}^{p}$ with $\mathcal{A}_{-\alpha q / p}^{q}\left(\mathbb{D}_{e}\right)$ by the duality

$$
\langle f, g\rangle_{e}=\left\langle f, R^{-1} g\right\rangle, \quad f \in \mathcal{D}_{\alpha}^{p}, g \in \mathcal{A}_{-\alpha q / p}^{q}\left(\mathbb{D}_{e}\right) .
$$

In the following we will introduce the tools to use the Hedenmalm and Shields Theorem [HS, Theorem 1]. For all $\varphi \in \mathcal{D}_{\alpha}^{p^{\prime}}$, we set

$$
\widetilde{\varphi}(\lambda)=\left\langle f_{\lambda}, \varphi\right\rangle, \quad \lambda \in \mathbb{D}_{e}
$$

where $f_{\lambda}$ is given by

$$
f_{\lambda}(z)=(\lambda-z)^{-1}, \quad z \in \mathbb{D} .
$$

We define then as in [HS]

Let $\varphi \in \mathcal{D}_{\alpha}^{p \prime}$, we have

$$
\mathcal{D}_{\alpha}^{p *}=\left\{\widetilde{\varphi}, \varphi \in \mathcal{D}_{\alpha}^{p \prime}\right\} .
$$

$$
\widetilde{\varphi}(\lambda)=\left\langle\sum_{n=0}^{\infty} \frac{z^{n}}{\lambda^{n+1}}, \varphi\right\rangle=\sum_{n=0}^{\infty} \frac{\left\langle z^{n}, \varphi\right\rangle}{\lambda^{n+1}} .
$$


We identify $\varphi$ as an element of $\mathcal{A}_{-\alpha q / p}^{q}(\mathbb{D})$ that we write

$$
\varphi(z)=\sum_{n \geq 0} a_{n} z^{n}, \quad z \in \mathbb{D}
$$

So if $n=0,\left\langle z^{n}, \varphi\right\rangle=\overline{\varphi(0)}=\overline{a_{0}}$ and if $n \geq 1$,

$$
\begin{aligned}
\left\langle z^{n}, \varphi\right\rangle & =\int_{\mathbb{D}} n z^{n-1} \overline{S^{*} \varphi(z)} d A(z) \\
& =\int_{\mathbb{D}} n z^{n-1} \sum_{m=1}^{\infty} \overline{a_{m}} \bar{z}^{m-1} d A(z) \\
& =\sum_{m=1}^{\infty} n \overline{a_{m}} \int_{0}^{1} \int_{0}^{2 \pi} r^{n+m-2} e^{i \theta(n-m)} d \theta / \pi r d r \\
& =\overline{a_{n}} \int_{0}^{1} 2 n r^{2 n-1} d r=\overline{a_{n}} .
\end{aligned}
$$

Thus for $\lambda \in \mathbb{D}_{e}$,

$$
\widetilde{\varphi}(\lambda)=\sum_{n=0}^{\infty} \frac{\overline{a_{n}}}{\lambda^{n+1}}
$$

Moreover, according to (2.2), we also have

$$
R \varphi(\lambda)=\sum_{n=0}^{\infty} \frac{\overline{a_{n}}}{\lambda^{n+1}}, \quad \lambda \in \mathbb{D}_{e}
$$

So

$$
\mathcal{D}_{\alpha}^{p *}=\mathcal{A}_{-\alpha q / p}^{q}\left(\mathbb{D}_{e}\right) .
$$

The following lemma will be useful for expressing duality (see [HS, Lemma 3]).

Lemma 2.3. Let $p>1$ and $-1<\alpha<p-1$. Let $f \in \mathcal{D}_{\alpha}^{p}$ and $g \in \mathcal{A}_{-\alpha q / p}^{q}\left(\mathbb{D}_{e}\right)$. For $0 \leq r<1$, we set

$$
f_{r}(z)=f(r z), \quad z \in \mathbb{D} \quad \text { and } \quad g_{1 / r}(z)=g(z / r), \quad z \in \mathbb{D}_{e}
$$

Then

$$
\langle f, g\rangle_{e}=\lim _{r \rightarrow 1^{-}}\left\langle f_{r}, g_{1 / r}\right\rangle_{e}=\lim _{r \rightarrow 1^{-}} \sum_{n=0}^{\infty} a_{n} b_{n} r^{n}=\lim _{r \rightarrow 1^{-}} \frac{1}{2 \pi} \int_{0}^{2 \pi} f\left(r e^{i \theta}\right) g\left(e^{i \theta} / r\right) e^{i \theta} d \theta
$$

where $f(z)=\sum_{n=0}^{\infty} a_{n} z^{n}$ and $g(1 / z)=\sum_{n=0}^{\infty} b_{n} z^{n+1}, z \in \mathbb{D}$. 


\section{CyCLICITY IN $\mathcal{D}_{\alpha}^{p}$}

We start this section by comparing the spaces $\mathcal{D}_{\alpha}^{p}$ and the Hardy spaces $H^{p}(\mathbb{D})$. We suppose $p \geq 1$ and $\alpha>-1$. Let $H^{\infty}(\mathbb{D})$ be the algebra of bounded analytic functions on the open unit disc $\mathbb{D}$ and let $H^{p}(\mathbb{D})$ be the Hardy space of analytic functions $f$ on $\mathbb{D}$ such that

$$
\|f\|_{H^{p}}=\sup _{r<1} M_{p}(f, r)<\infty
$$

where

$$
M_{p}(f, r)=\left(\frac{1}{2 \pi} \int_{0}^{2 \pi}\left|f\left(r e^{i \theta}\right)\right|^{p} d \theta\right)^{1 / p} .
$$

Let $\mathscr{N}$ be the Nevanlinna class of analytic functions $f$ on $\mathbb{D}$ for which

$$
\sup _{r<1} \int_{\mathbb{T}} \ln ^{+}|f(r \zeta)||d \zeta|<\infty .
$$

By Fatou's Theorem, the radial limit $f^{*}(\zeta)=\lim _{r \rightarrow 1-} f(r \zeta)$ exists a.e on $\mathbb{T}$ and $\ln \left|f^{*}\right| \in$ $L^{1}(\mathbb{T})$. Recall that $f \in \mathscr{N}$ if and only if $f=\varphi / \psi$, where $\varphi, \psi \in H^{\infty}(\mathbb{D})$.

Let $\mathscr{N}^{+}$be the Smirnov class of analytic functions $f \in \mathscr{N}$ such that

$$
\sup _{r<1} \int_{\mathbb{T}} \ln ^{+}|f(r \zeta)||d \zeta|=\int_{\mathbb{T}} \ln ^{+}\left|f^{*}(\zeta)\right||d \zeta| .
$$

The function $f \in \mathscr{N}^{+}$if and only if $f=\varphi / \psi$ where $\varphi, \psi \in H^{\infty}(\mathbb{D})$ and $\psi$ is an outer function, that is, $\psi$ has the form

$$
\psi(z)=\exp \int_{\mathbb{T}} \frac{\zeta+z}{\zeta-z} \log \psi^{*}(\zeta) \frac{|d \zeta|}{2 \pi}, \quad z \in \mathbb{D} .
$$

A function $f \in H^{p}(\mathbb{D})$ is cyclic for $H^{p}(\mathbb{D})$ if and only if $f$ is outer [N, 4.8.4]. We then study the different possible inclusions between the spaces $\mathcal{D}_{\alpha}^{p}$ and $H^{p}(\mathbb{D})$ to obtain first conditions on the cyclicity in the Dirichlet spaces.

Proposition 3.1. Let $p \geq 1$ and $\alpha>-1$. If $p<\alpha+1$ then $H^{p}(\mathbb{D})$ is continuously embedded in $\mathcal{D}_{\alpha}^{p}$. Consequently, if $f \in H^{p}(\mathbb{D})$ is outer then $f$ is cyclic for $\mathcal{D}_{\alpha}^{p}$.

Proof. Let $f \in H^{p}(\mathbb{D}), z=r e^{i t} \in \mathbb{D}$ and $r<\rho<1$. By Cauchy's formula,

$$
f^{\prime}(z)=\frac{1}{2 \pi} \int_{0}^{2 \pi} \frac{f\left(\rho e^{i(\theta+t)}\right)}{\left(\rho e^{i \theta}-r\right)^{2}} \rho e^{i(\theta-t)} d \theta .
$$


Now, by Minkowski's inequality,

$$
\begin{aligned}
M_{p}\left(f^{\prime}, r\right) & =\left(\frac{1}{2 \pi} \int_{0}^{2 \pi}\left|\frac{1}{2 \pi} \int_{0}^{2 \pi} \frac{f\left(\rho e^{i(\theta+t)}\right)}{\left(\rho e^{i \theta}-r\right)^{2}} \rho e^{i(\theta-t)} d \theta\right|^{p} d t\right)^{1 / p} \\
& \leq \frac{\rho}{2 \pi} \int_{0}^{2 \pi}\left(\frac{1}{2 \pi} \int_{0}^{2 \pi} \frac{\left|f\left(\rho e^{i(\theta+t)}\right)\right|^{p}}{\left|\rho e^{i \theta}-r\right|^{2 p}} d t\right)^{1 / p} d \theta \\
& \leq \frac{1}{2 \pi} \int_{0}^{2 \pi} \frac{\rho}{\left|\rho e^{i \theta}-r\right|^{2}} d \theta M_{p}(f, \rho) \\
& =\frac{\rho}{\rho^{2}-r^{2}} M_{p}(f, \rho) \leq \frac{1}{\rho-r} M_{p}(f, \rho) .
\end{aligned}
$$

Now letting $\rho \rightarrow 1$, we get

$$
\frac{1}{2 \pi} \int_{0}^{2 \pi}\left|f^{\prime}\left(r e^{i \theta}\right)\right|^{p} d \theta \leq \frac{1}{(1-r)^{p}}\|f\|_{H^{p}}^{p},
$$

Since $p<\alpha+1$,

$$
\begin{aligned}
\int_{\mathbb{D}}\left|f^{\prime}(z)\right|^{p} \mathrm{dA}_{\alpha}(z) & =\int_{0}^{1} \int_{0}^{2 \pi}\left|f^{\prime}\left(r e^{i \theta}\right)\right|^{p} d \theta\left(1-r^{2}\right)^{\alpha} r d r / \pi \\
& \leq 2^{\alpha+1} \int_{0}^{1} \frac{(1-r)^{\alpha}}{(1-r)^{p}} d r\|f\|_{H^{p}}^{p} \\
& =\frac{2^{\alpha+1}}{\alpha+1-p}\|f\|_{H^{p}}^{p}
\end{aligned}
$$

So $H^{p}(\mathbb{D})$ is continuously embedded in $\mathcal{D}_{\alpha}^{p}$. Now the result follows from the fact that an outer function is cyclic in $H^{p}(\mathbb{D})$.

Remark. If $p<\alpha+1$, the Dirichlet space $\mathcal{D}_{\alpha}^{p}=\mathcal{A}_{\alpha-p}^{p}(\mathbb{D})$, see $[\mathrm{Wu}]$. Therefore, in this case, there exists an inner function which is cyclic in $\mathcal{D}_{\alpha}^{p}$, see [Ro]. If $p>\alpha+1$ we have the following result.

Proposition 3.2. Let $p>1$ and $p>\alpha+1$. The Dirichlet space $\mathcal{D}_{\alpha}^{p}$ is continuously embedded in $H^{p}(\mathbb{D})$. Therefore if $f \in \mathcal{D}_{\alpha}^{p}$ is cyclic in $\mathcal{D}_{\alpha}^{p}$ then $f$ is an outer function.

Proof. Let $f \in \mathcal{D}_{\alpha}^{p}$ and $r \in[1 / 2,1[$. We have

$$
f\left(r e^{i \theta}\right)=\int_{0}^{r} f^{\prime}\left(s e^{i \theta}\right) e^{i \theta} d s+f(0) .
$$

Note that $|f(0)| \leq\|f\|_{\mathcal{D}_{\alpha}^{p}}$ and by subharmonicity, there exists $C>0$ such that $\left|f^{\prime}\left(s e^{i \theta}\right)\right| \leq$ $C\|f\|_{\mathcal{D}_{\alpha}^{p}}, 0 \leq s \leq 1 / 2$. So

$$
\left|f\left(r e^{i \theta}\right)\right| \leq \int_{1 / 2}^{r}\left|f^{\prime}\left(s e^{i \theta}\right)\right| d s+(C / 2+1)\|f\|_{\mathcal{D}_{\alpha}^{p}} .
$$


By Hölder's inequality, and since $\alpha q / p=\alpha /(p-1)<1$,

$$
\begin{gathered}
\left(\int_{0}^{2 \pi}\left|f\left(r e^{i \theta}\right)\right|^{p} d \theta\right)^{1 / p} \lesssim\left[\int_{0}^{2 \pi}\left(\int_{1 / 2}^{r}\left|f^{\prime}\left(s e^{i \theta}\right)\right| d s\right)^{p} d \theta\right]^{1 / p}+\|f\|_{\mathcal{D}_{\alpha}^{p}} \\
\lesssim\left[\int_{0}^{2 \pi}\left(\int_{1 / 2}^{r}\left|f^{\prime}\left(s e^{i \theta}\right)\right|^{p}\left(1-s^{2}\right)^{\alpha} d s\right)\left(\int_{1 / 2}^{r}\left(1-s^{2}\right)^{-\alpha q / p} d s\right)^{p / q} d \theta\right]^{1 / p}+\|f\|_{\mathcal{D}_{\alpha}^{p}} \\
\lesssim\left[\int_{0}^{2 \pi} \int_{1 / 2}^{1}\left|f^{\prime}\left(s e^{i \theta}\right)\right|^{p}\left(1-s^{2}\right)^{\alpha} 2 s d s d \theta\right]^{1 / p}\left[\int_{1 / 2}^{1}\left(1-s^{2}\right)^{-\alpha q / p} d s\right]^{1 / q}+\|f\|_{\mathcal{D}_{\alpha}^{p}} \\
\lesssim\left(1-\frac{\alpha q}{p}\right)\|f\|_{\mathcal{D}_{\alpha}^{p}}+\|f\|_{\mathcal{D}_{\alpha}^{p} .}
\end{gathered}
$$

So $\|f\|_{H^{p}} \lesssim\|f\|_{\mathcal{D}_{\alpha}^{p}}$. Hence if $f$ is cyclic for $\mathcal{D}_{\alpha}^{p}$ then $f$ is also cyclic for $H^{p}(\mathbb{D})$ and $f$ is then an outer function.

Remark. We have $\mathscr{D}_{1}^{2}(\mathbb{D})=H^{2}(\mathbb{D})$ and $\mathscr{D}_{0}^{2}(\mathbb{D})=\mathcal{D}$. So if $1 \leq p \leq 2$ and $p=\alpha+1$, we obtain by interpolation theorem $[\mathrm{Wu},(3.8)])$, that $\mathcal{D}_{\alpha}^{p}$ is continuously embedded in $H^{p}(\mathbb{D})$. Futhermore if $p>\alpha+2$, then $\mathcal{D}_{\alpha}^{p}$ is continuously embedded in $H^{\infty}(\mathbb{D})$ (see the proof of [Wu, Theorem 4.2]).

We can summarize here all the inclusions obtained:

$$
\begin{aligned}
p<\alpha+1 & \Longrightarrow H^{p}(\mathbb{D}) \subset \mathcal{D}_{\alpha}^{p}=\mathcal{A}_{\alpha-p}^{p}(\mathbb{D}) \\
1 \leq p \leq 2 \text { and } p=\alpha+1 & \Longrightarrow \mathcal{D}_{\alpha}^{p} \subset H^{p}(\mathbb{D}) \\
p>\alpha+1 & \Longrightarrow \mathcal{D}_{\alpha}^{p} \subset H^{p}(\mathbb{D}) \\
p>\alpha+2 & \Longrightarrow \mathcal{D}_{\alpha}^{p} \subset H^{\infty}(\mathbb{D})
\end{aligned}
$$

We assume in the following that $p>\alpha+1$. We will prove that any outer function of $A(\mathbb{D}) \cap \mathcal{D}_{\alpha}^{p}$ whose set of zeros is reduced to a single point is cyclic in $\mathcal{D}_{\alpha}^{p}$. For that we will use a Hedenmalm-Shields Theorem [HS, Theorem 1]. We first need to define the following notions. Let $X \subset \operatorname{Hol}(\mathbb{D})$ be a Banach space. The multiplier set of $X$, noted $M(X)$, is defined by

$$
M(X)=\{\varphi \in \operatorname{Hol}(\mathbb{D}), \varphi f \in X, \forall f \in X\} .
$$

If $X \subset \operatorname{Hol}\left(\mathbb{D}_{e}\right)$ we define in a similar way $M(X)$.

As in [HS] we identify the dual $X^{\prime}$ of $X$ with a space $X^{*}$ of holomorphic functions on $\mathbb{D}_{e}$. Finally for $E \subset \mathbb{T}$ a closed set of zero Lebesgue measure, we set

$$
\mathscr{H}_{E}\left(\mathscr{N}^{+}, X^{*}\right)=\left\{\varphi \in \operatorname{Hol}(\mathbb{C} \cup\{\infty\} \backslash E),\left.\varphi\right|_{\mathbb{D}} \in \mathscr{N}^{+}(\mathbb{D}),\left.\varphi\right|_{\mathbb{D}_{e}} \in X^{*}\right\} .
$$

We denote by $\operatorname{Hol}(\overline{\mathbb{D}})$, respectively $\operatorname{Hol}\left(\overline{\mathbb{D}_{e}}\right)$, the space of all holomorphic functions in a neighborhood of $\overline{\mathbb{D}}$, respectively $\overline{\mathbb{D}_{e}}$. 
Theorem 3.3 (Hedenmalm-Shields $[\mathrm{HS}]$ ). Let $X \subset \mathrm{Hol}(\mathbb{D})$ be a Banach space. Assume that

(1) The embedding map of $X$ into $\operatorname{Hol}(\overline{\mathbb{D}})$ is continuous and $X$ contains $\operatorname{Hol}(\overline{\mathbb{D}})$ as a dense subspace

(2) $X \cap A(\mathbb{D})$ is a Banach algebra, containing $\operatorname{Hol}(\overline{\mathbb{D}})$ as a dense algebra.

(3) $\operatorname{Hol}(\overline{\mathbb{D}}) \subset M(X)$.

(4) $\operatorname{Hol}\left(\overline{\mathbb{D}_{e}}\right) \subset M\left(X^{*}\right)=H^{\infty}\left(\mathbb{D}_{e}\right)$.

If $f \in X \cap A(\mathbb{D})$ is an outer function and if

$$
\mathscr{H}_{\mathcal{Z}(f)}\left(\mathscr{N}^{+}, X^{*}\right)=\{0\}
$$

then $f$ is cyclic in $X$.

Hedenmalm and Shields show that if $f \in A(\mathbb{D}) \cap \mathscr{D}_{0}^{2}(\mathbb{D})$ is an outer function and $\mathcal{Z}(f)=$ $\{1\}$ then $\mathscr{H}_{\mathcal{Z}(f)}\left(\mathscr{N}^{+}, \mathscr{D}_{0}^{2}(\mathbb{D})^{*}\right)=\{0\}$ and so $f$ is cyclic (see also [EKR2, EKMR]). We will prove a similar result for $\mathcal{D}_{\alpha}^{p}$ where $\alpha+1<p \leq \alpha+2$.

Theorem 3.4. Let $p>1$ and $p>\alpha+1$. If $f \in A(\mathbb{D}) \cap \mathcal{D}_{\alpha}^{p}$ is outer and if

$$
\mathscr{H}_{\mathcal{Z}(f)}\left(\mathscr{N}^{+}, \mathcal{D}_{\alpha}^{p *}\right)=\{0\}
$$

then $f$ is cyclic on $\mathcal{D}_{\alpha}^{p}$.

To prove this result, we will use Theorem 3.3. For that we need only to show the following lemma (see the proof of [DRS, lemma 11]).

Lemma 3.5. Let $p>1$ and $\alpha>-1$. Then $M\left(\mathcal{A}_{\alpha}^{p}\left(\mathbb{D}_{e}\right)\right)=H^{\infty}\left(\mathbb{D}_{e}\right)$.

Proof. Let $f \in \mathcal{A}_{\alpha}^{p}\left(\mathbb{D}_{e}\right)$ and $g \in H^{\infty}\left(\mathbb{D}_{e}\right)$. We have

$$
\int_{\mathbb{D}_{e}}|f(z) g(z)|^{p} \frac{\left(|z|^{2}-1\right)^{\alpha}}{|z|^{4-p+2 \alpha}} \mathrm{dA}(z) \leq\|g\|_{\infty}^{p}\|f\|_{\mathcal{A}_{\alpha}^{p}}^{p} .
$$

So $f g \in \mathcal{A}_{\alpha}^{p}\left(\mathbb{D}_{e}\right)$ and $H^{\infty}\left(\mathbb{D}_{e}\right) \subset M\left(\mathcal{A}_{\alpha}^{p}\left(\mathbb{D}_{e}\right)\right)$.

Now let $g \in M\left(\mathcal{A}_{\alpha}^{p}\left(\mathbb{D}_{e}\right)\right)$ and let $M_{g}: \mathcal{A}_{\alpha}^{p}\left(\mathbb{D}_{e}\right) \rightarrow \mathcal{A}_{\alpha}^{p}\left(\mathbb{D}_{e}\right)$ be the operator given by $M_{g}(f)=f g$. By the closed graph theorem, $M_{g}$ is bounded. For $z \in \mathbb{D}_{e}$, the linear functional $\Lambda_{z}: \mathcal{A}_{\alpha}^{p}\left(\mathbb{D}_{e}\right) \rightarrow \mathbb{C}$ defined by $\Lambda_{z}(f)=f(z)$, is continuous ([HKZ, proposition 1.1]). So for $f \in \mathcal{A}_{\alpha}^{p}\left(\mathbb{D}_{e}\right)$ and $z \in \mathbb{D}_{e}$,

$$
|f(z) g(z)|=\left|\Lambda_{z}\left(M_{g} f\right)\right| \leq\left\|\Lambda_{z}\right\|\left\|M_{g}\right\|\|f\|_{\mathcal{A}_{\alpha}^{p}} .
$$

Hence

$$
\left\|\Lambda_{z}\right\||g(z)| \leq\left\|\Lambda_{z}\right\|\left\|M_{g}\right\|
$$

and $g \in H^{\infty}\left(\mathbb{D}_{e}\right)$. So $M\left(\mathcal{A}_{\alpha}^{p}\left(\mathbb{D}_{e}\right)\right) \subset H^{\infty}\left(\mathbb{D}_{e}\right)$. On the other hand the inclusion $H^{\infty}\left(\mathbb{D}_{e}\right) \subset$ $M\left(\mathcal{A}_{\alpha}^{p}\left(\mathbb{D}_{e}\right)\right)$ is obvious.

By identifying the dual of $\mathcal{D}_{\alpha}^{p}$ with $\mathcal{A}_{-\alpha q / p}^{q}\left(\mathbb{D}_{e}\right)$, we have for $f \in \mathcal{D}_{\alpha}^{p}$ and $\varphi \in \mathcal{A}_{-\alpha q / p}^{q}\left(\mathbb{D}_{e}\right)$,

$$
\varphi \in\left([f]_{\mathbb{N}}^{\mathcal{D}_{\alpha}^{p}}\right)^{\perp} \Longleftrightarrow\left\langle z^{n} f, \varphi\right\rangle_{e}=0, \quad \forall n \in \mathbb{N} .
$$


Lemma 3.6. Let $p>1$ and $p>\alpha+1$. Let $E \subset \mathbb{T}$ a closed set of Lebesgue measure, $\varphi \in \mathscr{H}_{E}\left(\mathscr{N}^{+}, \mathcal{D}_{\alpha}^{p *}\right)$ and $f \in \mathcal{D}_{\alpha}^{p}$. If the family of functions

$$
z \in \mathbb{T} \mapsto f(r z) \varphi(z / r), \quad 1 / 2<r<1
$$

is uniformly integrable on $\mathbb{T}$, then $\varphi \in\left([f]_{\mathbb{N}}^{\mathcal{D}_{\alpha}^{p}}\right)^{\perp}$.

Proof. This result holds by using the analogue arguments like those in [EKR2, Lemma 3.4] for the classical Dirichlet space. For the sake of completeness, we include it here. Let $f \in \mathcal{D}_{\alpha}^{p}$ and $\left.\varphi\right|_{\mathbb{D}_{e}} \in \mathcal{D}_{\alpha}^{p *}=\mathcal{A}_{-\alpha q / p}^{q}\left(\mathbb{D}_{e}\right)$. By Proposition 2.3, we have

$$
\langle f, \varphi\rangle=\lim _{r \rightarrow 1^{-}} \frac{1}{2 \pi} \int_{0}^{2 \pi} f\left(r e^{i \theta}\right) \varphi\left(e^{i \theta} / r\right) e^{i \theta} d \theta .
$$

By Proposition 3.2, $\mathcal{D}_{\alpha}^{p} \subset H^{p}(\mathbb{D})$ and so $f^{*}$, the radial limit of $f$, exists a.e. on $\mathbb{T}$. Since $\varphi \in \operatorname{Hol}(\mathbb{C} \backslash E)$ and $E$ is a closed set of Lebesgue measure zero, $\varphi(z / r) \longrightarrow \varphi(z)$ exists a.e on $\mathbb{T}$ when $r \rightarrow 1^{-}$. So the family of the functions $z \mapsto f(r z) \varphi(z / r)$ converges a.e to $f^{*} \varphi$ when $r \rightarrow 1^{-}$. By uniform integrability, this family of functions converges in $L^{1}(\mathbb{T})$. Then

$$
\langle f, \varphi\rangle=\frac{1}{2 \pi} \int_{0}^{2 \pi} f^{*}\left(e^{i \theta}\right) \varphi\left(e^{i \theta}\right) e^{i \theta} d \theta
$$

Futhermore $\varphi \in \mathscr{N}^{+}$and $f \in H^{p}(\mathbb{D}) \subset \mathscr{N}^{+}$, so then $f \varphi \in \mathscr{N}^{+}$. Since the radial limit $(f \varphi)^{*}=f^{*} \varphi \in L^{1}(\mathbb{T})$, by Smirnov's generalized maximum principal [D, Theorem 2.11], $f \phi \in H^{1}(\mathbb{D})$ and so $\widehat{f^{*} \varphi}(n)=0$ :

$$
\widehat{f^{*} \varphi}(n)=\langle f, \varphi\rangle=\frac{1}{2 \pi} \int_{0}^{2 \pi} f^{*}\left(e^{i \theta}\right) \varphi\left(e^{i \theta}\right) e^{i \theta} d \theta=0 .
$$

Repeating the same argument with $f$ replaced by $z^{n} f$, we get $\left\langle z^{n} f, \varphi\right\rangle=0$ for all $n \in \mathbb{N}$.

We have the following classical Lemma

Lemma 3.7. Let $p>1$ and $p>\alpha+1$. Let $E \subset \mathbb{T}$ be a closed set of Lebesgue measure zero and $\varphi \in \mathscr{H}_{E}\left(\mathscr{N}^{+}, \mathcal{D}_{\alpha}^{p *}\right)$. Then there exists a constant $C>0$ such that

$$
|\varphi(z)| \leq \frac{C}{\operatorname{dist}(z, E)^{4}}, \quad 1<|z|<2
$$

Proof. Let $\varphi \in \mathscr{H}_{E}\left(\mathscr{N}^{+}, \mathcal{D}_{\alpha}^{p *}\right)$. Since $\left.\varphi\right|_{\mathbb{D}} \in \mathscr{N}^{+},\left.\varphi\right|_{\mathbb{D}}=\varphi_{i} \varphi_{o}$, where $\varphi_{i}$ is an inner function and $\varphi_{o}$ is an outer function in $\mathscr{N}$ (see [D, p. 25]). Futhermore, since $E$ has Lebesgue measure zero , $\varphi(z)=\varphi^{*}(z)=\lim _{r \rightarrow 1^{-}} \varphi(r z)$ exists a.e on $\mathbb{T}$. The function 
$\log |\varphi|$ being in $L^{1}(\mathbb{T})$, we get

$$
\begin{aligned}
|\varphi(z)| & \leq\left|\varphi_{o}(z)\right|=\left|\exp \left(\frac{1}{2 \pi} \int_{0}^{2 \pi} \frac{e^{i t}+z}{e^{i t}-z} \log \left|\varphi\left(e^{i t}\right)\right| d t\right)\right| \\
& \leq \exp \left(\frac{1}{2 \pi} \int_{0}^{2 \pi} \frac{1-|z|^{2}}{\left|e^{i t}-z\right|^{2}} \log \left|\varphi\left(e^{i t}\right)\right| d t\right) \\
& \leq \exp \left(\frac{1-|z|^{2}}{(1-|z|)^{2}} \int_{0}^{2 \pi}|\log | \varphi\left(e^{i t}\right)|| d t\right) \\
& \leq \exp \left(\frac{2}{1-|z|}\|\log |\varphi|\|_{L^{1}(\mathbb{T})}\right) \\
& \leq \exp \left(\frac{C_{1}}{1-|z|}\right)
\end{aligned}
$$

for some constant $C_{1}>0$. Let $z \in \mathbb{D}_{e}$ with $|z| \leq 2$. The disc of radius $(|z|-1) / 2$ centered at $z, D(z,(|z|-1) / 2)$ is contained in $\mathbb{D}_{e}$. Since $\left.\varphi\right|_{\mathbb{D}_{e}} \in \mathcal{A}_{-\alpha q / p}^{q}\left(\mathbb{D}_{e}\right)$, by subharmonicity of $|\varphi|$ and for $q=p /(p-1) \geq 1$, we obtain

$$
\begin{aligned}
\frac{(|z|-1)^{2}}{4}|\varphi(z)|^{q} & \leq \frac{1}{\pi} \int_{D(z,(|z|-1) / 2)}|\varphi(w)|^{q} d A(w) \\
& \leq \frac{1}{\pi} \int_{D(z,(|z|-1) / 2)}|\varphi(w)|^{q} \frac{\left(|w|^{2}-1\right)^{-\alpha q / p}}{|w|^{4-q-2 \alpha q / p}} \frac{|w|^{4-q-2 \alpha q / p}}{\left(|w|^{2}-1\right)^{-\alpha q / p}} d A(w) \\
& \leq \max \left(2^{2 \alpha q / p}, 2^{4-q}\right) \int_{\mathbb{D}_{e}}|\varphi(w)|^{q} \frac{\left(|w|^{2}-1\right)^{-\alpha q / p}}{|w|^{4-q-2 \alpha q / p}} d A(w) \\
& \leq \max \left(2^{2 \alpha q / p}, 2^{4-q}\right)\left\|\left.\varphi\right|_{\mathbb{D}_{e}}\right\|_{\mathcal{B}_{-\alpha q / p}^{q}} .
\end{aligned}
$$

So

$$
|\varphi(z)| \leq \frac{C_{2}}{(|z|-1)^{2}}, \quad 1<|z| \leq 2,
$$

for some constant $C_{2}>0$. Since $\log |\varphi|$ is subharmonic function, by Taylor-Williams estimates [RW, lemma 5.8 and 5.9] and [EKMR, Lemma 9.6.5], we get the lemma.

The following result allows us to reduce the study of cyclic vectors vanishing on a closed set $E$ to the study of cyclicity of particular functions. More precisely we have

Theorem 3.8. Let $p>1$ and $p>\alpha+1$. Let $f \in \mathcal{D}_{\alpha}^{p}$ and $E \subset \mathbb{T}$ be a closed set of Lebesgue measure zero. If there exists a constant $C_{1}>0$ such that,

$$
|f(z)| \leq C_{1} \operatorname{dist}(z, E)^{4}, \quad z \in \mathbb{D},
$$

then

$$
\mathscr{H}_{E}\left(\mathscr{N}^{+}, \mathcal{D}_{\alpha}^{p *}\right) \subset\left([f]_{\mathbb{N}}^{\mathcal{D}_{\alpha}^{p}}\right)^{\perp}
$$


This means that for all $g \in \mathscr{H}_{E}\left(\mathscr{N}^{+}, \mathcal{D}_{\alpha}^{p *}\right),\left.g\right|_{\mathbb{D}_{e}} \in\left([f]_{\mathbb{N}}^{\mathcal{D}_{\alpha}^{p}}\right)^{\perp}$ i.e.

$$
\left\langle z^{n} f,\left.g\right|_{\mathbb{D}_{e}}\right\rangle_{e}=0, \quad \forall n \in \mathbb{N} .
$$

Proof. Let $\varphi \in \mathscr{H}_{E}\left(\mathscr{N}^{+}, \mathcal{D}_{\alpha}^{p *}\right)$. By Lemma 3.7, there exists a constant $C_{2}>0$ such that

$$
|\varphi(z)| \leq \frac{C_{2}}{\operatorname{dist}(z, E)^{4}}, \quad 1<|z|<2
$$

So for $1 / 2<r<1$ and $z \in \mathbb{T}$, we have

$$
|f(r z) \varphi(z / r)| \leq C_{1} C_{2} \frac{\operatorname{dist}(r z, E)^{4}}{\operatorname{dist}(z / r, E)^{4}} \leq C_{1} C_{2} .
$$

The family of the functions $z \mapsto f(r z) \varphi(z / r)$ is uniformly integrable on $\mathbb{T}$ for $1 / 2<r<1$, thus by Lemma 3.6, $\varphi \in\left([f]_{\mathbb{N}}^{\mathcal{D}_{\alpha}^{p}}\right)^{\perp}$, which finishes the proof.

Corollary 3.9. Let $p>1$ such that $\alpha+1<p \leq \alpha+2$. We have

$$
\mathscr{H}_{\{1\}}\left(\mathscr{N}^{+}, \mathcal{D}_{\alpha}^{p *}\right)=\{0\} .
$$

Proof. Let $f(z):=(z-1)^{4}$. We have $f \in \mathcal{D}_{\alpha}^{p}$ and $|f(z)| \leq|z-1|^{4}$. By Theorem 3.8 ,

$$
\mathscr{H}_{\{1\}}\left(\mathscr{N}^{+}, \mathcal{D}_{\alpha}^{p *}\right) \subset\left([f]_{\mathbb{N}}^{\mathcal{D}_{\alpha}^{p}}\right)^{\perp} .
$$

It suffices to prove that $f$ is cyclic. Let $\varphi \in \mathcal{A}_{-\alpha q / p}^{q}(\mathbb{D})$ such that

$$
\left\langle z^{n}(z-1), \varphi\right\rangle=0, \quad \forall n \in \mathbb{N} .
$$

Write $\varphi(z)=\sum_{n \geq 0} a_{n} z^{n}$, we get by $(2.4)$,

$$
\overline{a_{n}}=\left\langle z^{n}, \varphi\right\rangle=\left\langle z^{n+1}, \varphi\right\rangle=\overline{a_{n+1}} .
$$

Then

$$
\varphi(z)=\sum_{n=0}^{\infty} a_{n} z^{n}=\frac{a_{0}}{1-z}, \quad z \in \mathbb{D} .
$$

Suppose that $\varphi \neq 0$. Since $\varphi \in \mathcal{A}_{-\alpha q / p}^{q}(\mathbb{D})$, we have

$$
\int_{\mathbb{D}} \frac{\left(1-|z|^{2}\right)^{-\alpha q / p}}{|1-z|^{q}} \mathrm{dA}(z)<\infty,
$$

and so $q+\alpha q / p<2$ (see [HKZ, Theorem 1.7]), which contradicts the assumptions on $p$ and $\alpha$. So $\varphi=0$ and $[z-1]_{\mathbb{N}}^{\mathcal{D}_{\alpha}^{p}}=\mathcal{D}_{\alpha}^{p}$. In particular $z-1 \in\left[(z-1)^{2}\right]_{\mathbb{N}}^{\mathcal{D}_{\alpha}^{p}}$ and then

$$
\left[(z-1)^{2}\right]_{\mathbb{N}}^{\mathcal{D}_{\alpha}^{p}}=[z-1]_{\mathbb{N}}^{\mathcal{D}_{\alpha}^{p}}=\mathcal{D}_{\alpha}^{p} .
$$

With the same argument we obtain

$$
\left[(z-1)^{4}\right]_{\mathbb{N}}^{\mathcal{D}_{\alpha}^{p}}=\mathcal{D}_{\alpha}^{p}
$$

and $f(z)=(z-1)^{4}$ is cyclic in $\mathcal{D}_{\alpha}^{p}$. 
Remark. The proof of the previous result also gives us that for $p>\alpha+2$, the function $f(z)=z-1$ is not cyclic in $\mathcal{D}_{\alpha}^{p}$. Indeed by $(3.1), \varphi(z)=1 /(1-z) \in \mathcal{A}_{-\alpha q / p}^{q}(\mathbb{D})$ and $\varphi \perp z^{n} f, n \in \mathbb{N}$. More generally if $f \in A(\mathbb{D}) \cap \mathcal{D}_{\alpha}^{p}$ with $f(1)=0$, then $f$ is not cyclic in $\mathcal{D}_{\alpha}^{p}$. Indeed for $p>\alpha+2$, we have $\mathcal{D}_{\alpha}^{p} \subset H^{\infty}(\mathbb{D})$ with $\|\cdot\|_{H^{\infty}} \lesssim\|\cdot\|_{\mathcal{D}_{\alpha}^{p}}$ which implies

$$
[f]_{\mathbb{N}}^{\mathcal{D}_{\alpha}^{p}} \subset\{g \in A(\mathbb{D}), g(1)=0\} .
$$

Theorem 3.10. Let $p>1$ such that $\alpha+1<p \leq \alpha+2$ and let $f \in A(\mathbb{D}) \cap \mathcal{D}_{\alpha}^{p}$. If $f$ is an outer function and $\mathcal{Z}(f)$ is countable then $f$ is cyclic in $\mathcal{D}_{\alpha}^{p}$.

Proof. Since $\mathcal{Z}(f)$ is countable, by [BS, Theorem 3] it suffices to prove the theorem when the zero set is reduced to a single point. The result now follows by Theorem 3.4 and Corollary 3.9.

Acknowledgement. The authors thank Javad Mashreghi and Laval University for its support and hospitality. We also thank the referee for their comments and suggestions.

\section{REFERENCES}

[B] A. Beurling, Ensembles exceptionnels, Acta Math. 72 (1940), 1-13.

[BS] L. Brown, A. Shields, Cyclic vectors in the Dirichlet space, Trans. Amer. Math. Soc. 285 (1984), 269-304.

[C] L CARLESON Sets of uniqueness for functions regular in the unit circle, Acta Math. 87 (1952), 325345.

[D] P. L. Duren, Theory of Hp spaces, New York and London: Academic Press (1970).

[DRS] P. L. Duren, B. W. Romberg, A. L. Shields, Linear functionals on $H^{p}$ spaces with $0<p<1$, J. Reine Angew. Math. 238 (1969), 32-60.

[EKMR] O. El-Fallah, K. Kellay, J. Mashreghi, T. Ransford, A primer on the Dirichlet spaces, Cambridge Tracts in Mathematics 203. (2014).

[EKR1] O.El-Fallah, K.Kellay, T.Ransford, Cyclicity in the Dirichlet space, Ark. Mat. 44 (2006), no. $1,61-86$.

[EKR2] O. El-Fallah, K. Kellay, T. Ransford, On the Brown-Shields conjecture for the cyclicity in the Dirichlet space. Adv. Math. 222 (2009), No. 6, 2196-2214.

[EKR3] O. El-Fallah, K. Kellay, T. Ransford, Cantor sets and cyclicity in weighted Dirichlet spaces. J. Math. Anal. Appl. (2010) 372, No. 2, 565-573.

[G] J. Garnett, Bounded analytic functions, Vol. 236. Springer Science (2007).

[HS] H. Hedenmalm, A. Shields, Invariant subspaces in Banach spaces of analytic functions, Michigan Math. J, vol. 37, no 1, (1990), 91-104.

[HKZ] H. Hedenmalm; B. Korenblum; K. Zhu, Theory of Bergman spaces. Graduate Texts in Mathematics, 199. Springer-Verlag, New York, 2000.

[N] N. K. Nikolski, Operators, Functions, and Systems : An Easy Reading, Mathematical Surveys and Monographs, Volume 92.

[RS1] S. Richter, C. Sundberg, Invariant subspaces of the Dirichlet shift and pseudocontinuations, Trans. Amer. Math. Soc. 341 (1994), 863-879.

[Ro] J. W. RoBerts, Cyclic inner functions in the Bergman spaces and weak outer functions in $H^{p}$, $0<p<1$, Illinois J. of Math. 29, no. 1 (1985): 25-38.

[RW] B. A. TAYlor, D. L. Williams, Ideals in rings of analytic functions with smooth boundary values, Can. J. Math., Vol XXII, No. 6, (1970) 1266-1283.

[Wu] Z. Wu, Carleson Measure ans multipliers for Dirichlet Spaces, J. Funct. Anal. 169, (1999), $148-163$. 
Univ. Bordeaux, CNRS, Bordeaux INP, IMB, UMr 5251, F-33405 Talence, France.

E-mail address: kkellay@math.u-bordeaux.fr

E-mail address: florian.le-manach@math.u-bordeaux.fr

E-mail address: mohamed.zarrabi@math.u-bordeaux.fr 